\title{
The Role of Cardiopulmonary Bypass on the Early Postoperative IgG levels, Effect on the Postoperative Outcome in Cardiac Surgery Patients - A Pilot Study
}

\author{
Panagiotis Artemiou ${ }^{1}$, Stefania Mizakova ${ }^{1}$, Stefan Lukacin ${ }^{1}$, loannis Pashalidis ${ }^{2}$ \\ ${ }^{1}$ Dept. of Cardiovascular Surgery, University of P. J. Safarik, VUSCH, Kosice, Slovakia, ${ }^{2}$ Department \\ of Chemistry, University of Cyprus, Nicosia, Cyprus.
}

\begin{abstract}
Key words:
Postoperative

complications,

Immunoglobulin

$G$,

Cardiopulmonary

bypass, Cardiac

surgery

Abstract:

Objective: This study tried to elucidate the role of the cardiopulmonary bypass on the early postoperative immunoglobulin $G$ levels and any probable effects on the postoperative outcome of the patients.

Methods: 99 consecutive patients were studied. The evening after surgery the level of the immunoglobulin $G$ was obtained. The postoperative course of each patient during the first three days was followed. One way ANOVA was used for statistical analysis.

Results: 20 (20.2\%) patients had decreased early postoperative immunoglobulin G levels. 87 (87.87\%) patients were operated with cardiopulmonary bypass and 17 (19.54\%) of them had lower levels of postoperative immunoglobulin $G$ without any significantly increased clinically adverse events. The statistical analysis between the two groups included: pulmonary infiltrations, leukocytosis $>15 \times 10^{3} / \mathrm{mm}^{3}$, pulmonary dysfunction, mechanical ventilation $>24 \mathrm{~h}$, renal and hepatic dysfunction, postoperative ileus, postoperative delirium, sternal wound infection, thrombocytopenia $<60 \times 10^{3} /$ $\mathrm{mm}^{3}$ and sepsis. The statistical results were: $\mathrm{p}=0.815, \mathrm{p}=0.88, \mathrm{p}=0.93, \mathrm{p}=0.30, \mathrm{p}=0.67, \mathrm{p}=0.13, \mathrm{p}=0.84$, $\mathrm{p}=0.38, \mathrm{p}=0.76$ respectively.

Conclusion: In this pilot study we tried to explore the role of cardiopulmonary bypass on the early postoperative levels of immunoglobulin $G$ and to establish any correlation with the postoperative outcome. The use of cardiopulmonary bypass and its duration did not prove to be risk factors for low early postoperative immunoglobulin $G$ levels. In our opinion the prophylactic use of IVIG in this group of patients has no benefit.
\end{abstract}

(Cardiovasc. j. 2015; 7(2): 79-84)

\section{Introduction:}

Many postoperative complications after cardiac surgery are associated with some degree of immune dysfunction. ${ }^{1}$ In cardiac surgery, the use of cardiopulmonary bypass (CPB) results in some patients in a decrease in the postoperative levels of immunoglobulin G (IgG) ${ }^{2-4}$ Therefore the objectives of this study were to elucidate the role of the CPB on the early postoperative IgG levels and to study the probable effects on the postoperative outcome of the patients.

\section{Methods:}

\section{Study Population}

In this study, 99 consecutive patients were included, that were operated in our institution.
The patients had elective or urgent operations with or without CPB. Emergency patients were excluded from the study and patients with congenital or acquired immune deficiency as well.

\section{Management Protocol}

In the evening after surgery, the level of the IgG was obtained. The patient's characteristics that were analyzed were age, gender, Euroscore II value, CPB time, type of the operation, reexploration for bleeding and transfusion of more than four units of red pack cells.

With each patient the postoperative course during the first three days was followed and the incidence of the following postoperative complications was studied: 
a) pulmonary infiltrates on chest X-ray, b) leukocytosis $>15 \times 10^{3} / \mathrm{mm}^{3}$, c) pulmonary dysfunction defined as work of breathing more than moderate and oxygen requirements more than $50 \%-70 \%$, d) mechanical ventilation more than 24 hours, e) septic shock defined as mean blood pressure $<70 \mathrm{mmHg}$, cardiac index $>31 / \mathrm{m}^{2}$ and the use of high doses of inotropes, f) renal dysfunction defined as an increase in postoperative creatinine two times the baseline, g) hepatic dysfunction defined as an increase in postoperative hepatic enzymes and bilirubin, $\mathrm{h}$ ) gastrointestinal ileus diagnosed on abdominal X-ray, i) severe postoperative thrombocytopenia $<60 \times 10^{3} / \mathrm{mm}^{3}$, j) postoperative delirium requiring pharmacologic therapy and $\mathrm{k}$ ) postoperative sternal wound infections.

\section{Determination of serum IgG levels}

The levels of the IgG were determined by the method of immunoturbidimetry. The monoclonal and polyclonal antibodies were obtained from Diasys Diagnostic Systems GmbH (Holzheim GER). Levels less than $7 \mathrm{~g} / \mathrm{l}$ were considered low according to our laboratory reference value.

\section{Statistical analysis}

The data are presented as mean values +/- standard deviation (SD). One way ANOVA was used for statistical analysis to compare the various groups of variables. Student's $t$ and Tukey-Kramer test were used for $p$ calculation. Values of $p<0.05$ were considered significant. For the statistical analysis the software of JMP (Version 4, SAS Institute Inc, Cary, NC) was used.

\section{Results:}

A total of 99 consecutive patients were included in this study. $72(72.7 \%)$ patients were men and the rest $27(27.3 \%)$ were women. The average age was $63.84 \pm 11.56$ years old. The patients' characteristics are shown in table I.

Table-I

Baseline characteristics of study population

\begin{tabular}{ll}
\hline Characteristics & Number $(\%)(\mathrm{n}=99)$ \\
\hline Male & $72(72.7 \%)$ \\
Female & $27(27.3 \%)$ \\
Age & $63.84 \pm 11.56$ years \\
Euroscore II & $2.39 \% \pm 2.52 \%$ \\
CABG & $56(56.6 \%)$ \\
AVR & $36(36.4 \%)$ \\
MVR & $13(13.1 \%)$ \\
OPCAB & $12(12.1 \%)$ \\
Transfusions $>4$ RPC & $17(16.83 \%)$ \\
Re-exploration & $4(4 \%)$ \\
\hline
\end{tabular}

The mean Euroscore II value was $2.39 \% \pm 2.52 \%$. $56(56.6 \%)$ patients had coronary artery bypass graft (CABG), 36 (36.4\%) patients had aortic valve replacement (AVR), 13 (13.1\%) patients had mitral valve replacement (MVR) and 12 (12.1\%) patients underwent off-pump coronary artery bypass (OPCAB). 17 (17.2\%) patients received more than 4 units of red pack cells (RPC) and 4 (4\%) patients had re-exploration due to postoperative bleeding.

As far as the incidence of the postoperative complications are concerned, 13 (13.1\%) patients had pulmonary infiltrates diagnosed on chest Xray, $36(36.4 \%)$ patients had leukocytosis $>15 \times 10^{3} /$ $\mathrm{mm}^{3}, 10(10.1 \%)$ patients had pulmonary dysfunction, 1 (1\%) patient had mechanical ventilation more than 24 hours, 20 (20.2\%) patients had renal dysfunction, $23(23.2 \%)$ patients had hepatic dysfunction, $2(2.2 \%)$ patients had gastrointestinal ileus diagnosed on abdominal Xray, $16(16.2 \%)$ patients had postoperative delirium which required pharmacologic treatment, 6 (6.1\%) patients had sternal wound infection. Finally, no patient developed severe thrombocytopenia $<60 \times 10^{3} / \mathrm{mm}^{3}$ or sepsis.

From the 99 patients 79 (79.79\%) had normal (above $7 \mathrm{~g} / \mathrm{l})$ and the rest $20(20.2 \%)$ had decreased early postoperative IgG levels. 87 (87.87\%) patients were operated with CPB (CABG, AVR, MVR) and 17 (19.54\%) of them had low early postoperative IgG levels. $12(12.12 \%)$ patients had an OPCAB operation and $2(16.67 \%)$ of them had low early postoperative IgG levels. The mean IgG levels were $8.44 \pm 0.25 \mathrm{~g} / \mathrm{l}$. In the group with the early low $\mathrm{IgG}$ levels the mean level was $5.51 \pm 1.32 \mathrm{~g} / \mathrm{l}$.

Table II shows the statistical analysis between the two groups of patients with normal and low early IgG levels, the patients' characteristics and the incidence of the various postoperative complications.

The results showed that the levels of the early postoperative IgG levels had no significant effect on the postoperative outcome of the patients. The postoperative complications that were analyzed were: pulmonary infiltrations on chest X-ray, leukocytosis $>15 \times 10^{3} / \mathrm{mm}^{3}$, pulmonary dysfunction, mechanical ventilation $>24 \mathrm{~h}$, renal and hepatic dysfunction, postoperative gastrointestinal ileus, postoperative delirium, sternal wound infection, severe thrombocytopenia 
$<60 \times 10^{3} / \mathrm{mm}^{3}$ and sepsis. The statistical results were $p=0.81, p=0.88, p=0.93, p=0.30, p=0.67$, $p=0.13, p=0.84, p=0.38$ and $p=0.76$ respectively.

The patients were divided into three groups according to the Euroscore II value, low-risk (Euroscore II value <1.6\%), moderate risk (Euroscore II value 1.6\%-6.7\%) and high risk (Euroscore II value $>6.7 \%$ ) patients and the role of the Euroscore II value as a risk factor for low early postoperative IgG was analyzed. The statistical result was non-significant $(p=0.44)$. The results are shown in table III.

Moreover, the role of the use of CPB also as a risk factor for low early postoperative IgG levels was studied. 87 patients were operated with cardiopulmonary bypass and 12 patients had an OPCAB operation. The statistical result was nonsignificant $(p=0.60)$. The results are shown in table III.

The patients were also divided into two groups according the CPB time and its role as a risk factor for early low postoperative IgG levels was studied. In the first group the CPB was less than 60 minutes and on the second group the CPB time was more than 60 minutes. The statistical result was not significant $(p=0.55)$. The results are shown in table III.

Table-II

Postoperative IgG levels in relation to the patients characteristics and the incidence of the postoperative complications.

\begin{tabular}{lcccc}
\hline Characteristics & No(n=99) & $\begin{array}{c}\text { Norma } \\
\text { l IgG levels (n=99) }\end{array}$ & $\begin{array}{c}\text { Decreased } \\
\text { IgG levels (n=99) }\end{array}$ & $p$ value \\
\hline Male & 72 & 59 & 13 & 0.75 \\
Female & 27 & 20 & 7 & 0.75 \\
CABG & 56 & 42 & 14 & 0.93 \\
AVR & 36 & 33 & 3 & 0.09 \\
MVR & 13 & 11 & 2 & 0.29 \\
OPCAB & 12 & 10 & 2 & 0.63 \\
Pul. Infiltrates & 13 & 10 & 3 & 0.81 \\
Leukocytosis $>15 \times 10^{3}$ & 36 & 26 & 10 & 0.88 \\
Pul. Dysfunction & 10 & 6 & 4 & 0.93 \\
Mech. Ventilation $>24 h$ & 1 & 1 & 0 & 0.30 \\
Renal dysfunction & 20 & 16 & 4 & 0.67 \\
Hepatic dysfunction & 23 & 20 & 1 & 0.13 \\
Ileus on abd. X-ray & 2 & 1 & 2 & 0.84 \\
Delirium & 16 & 14 & 1 & 0.38 \\
Sternal wound infection & 6 & 5 & & 0.76 \\
\hline
\end{tabular}

Table-III

Postoperative IgG levels in relation to the Euroscore II value, the use of CPB and the CPB time.

\begin{tabular}{lcccc}
\hline & No.(n=99) & $\begin{array}{c}\text { Normal } \\
\text { IgG levels(n=99) }\end{array}$ & $\begin{array}{c}\text { Decreased } \\
\text { IgG levels(n=99) }\end{array}$ & $p$ value \\
\hline Low risk Euroscore II & 50 & 41 & 9 & 0.44 \\
Mod. risk Euroscore II & 45 & 35 & 10 & 0.44 \\
High risk Euroscore II & 4 & 3 & 1 & 0.44 \\
Use of CPB & 87 & 69 & 18 & 0.60 \\
No use of CPB & 12 & 10 & 2 & 0.60 \\
CPB $>60$ min & 60 & 49 & 11 & 0.55 \\
CPB $<60$ min & 27 & 20 & 7 & 0.55 \\
\hline
\end{tabular}




\section{Discussion:}

Cardiopulmonary bypass is known to have an immunosuppressive effect which includes lower postoperative immunoglobulin levels. The goal of our study was to examine the role of CPB on the early postoperative levels of IgG. $19.54 \%$ of the patients that were electively or semi-electively operated with CPB had decreased early postoperative IgG levels. The use of CPB and its duration did not prove to be risk factors for low early postoperative IgG levels. In this study we also tried to correlate this decreased early postoperative IgG levels with the postoperative outcome of the patients. Low early IgG levels did not correlate with survival or any other increased incidence of postoperative complications. Moreover $16.67 \%$ of the patients, who had an OPCAB operation, also had decreased early postoperative IgG levels which are assumed to be the result of the immunosuppressive effect of trauma and stress.

Similar results in the literature were presented by other authors. Firstly Lante et al. in their group of 83 patients who had elective cardiac operation studied the effects of CPB on the perioperative immunoglobulin levels. ${ }^{2}$ They showed that both IgG and IgM levels decreased significantly on postoperative day 1 while IgM levels returned to baseline on postoperative day 5, IgG levels remained below baseline even after postoperative day 5. Interestingly in their study there was an increase of Immunoglobulin E ( $\operatorname{IgE}$ ) levels as a result of the enhanced humoral immune response by the CPB.

Secondly, another older study by Siminelakis et al. quantified the immunosuppressive effect of CPB in a group of 20 consecutive patients and showed that also there is a postoperative reduction of the levels of IgG and IgA. ${ }^{3}$ The authors concluded that the observed immune alterations were mostly of no immunologic origin and were related to hemodilution and inflammation together with an immunosuppressive effect of trauma and stress.

The aim of our study was not to identify the mechanisms responsible for the postoperative immunosuppression of patients undergoing cardiac surgery. Szerafin et al. demonstrated that CABG surgery induces a massive long-lasting secretion of ST2 which is a protein related to immune suppression. ${ }^{4}$
Concerning the postoperative outcome of the patients, low early IgG levels did not correlate with survival or any other increased incidence of postoperative complications. Similar finding was described by Lante et al., who stated that the transient deficit in IgM and IgG levels found in their study did not result in any clinically adverse events and any therapeutic intervention appeared not to be required. ${ }^{2}$

Another goal of the study was to examine, if there was a group of patients who could be identified by low early IgG for complications and if there would be benefit from receiving hyperimmune gammaglobulin. In our study low early IgG levels did not correlate with survival or any other significant increased incidence of postoperative complication, so any prophylactic intervention with hyperimmune gammaglobulin appeared not to be required. According to the literature, the current experience is mainly based with septic patients despite the difference of the inflammatory reaction from sepsis and cardiopulmonary bypass with the second one being more predictable and selflimiting. Concerning this issue, many authors reported mixed results.

Rankin et al. identified a group of patients in their study that were elderly with significant comorbidity who were hospitalized with acute illness and were exhibiting nutritional and potentially immunologic depletion on admission..$^{5}$ This group developed an antibiotic-refractory pneumonia combined in some of the patients with renal failure. These patients were associated with low postoperative IgG levels. They received a high-dose intravenous immunoglobulin therapy (IVIG) and there was an improvement in clinical course over the subsequent 5 days. On the contrary, in the Early Supplemental Severe SIRS Treatment with IVIG in Score-Identified High-Risk Patients After Cardiac Surgery (ESSICS) study, patients who developed severe systemic inflammatory response syndrome (SIRS) received lower doses of IVIG without any improvement in short-term morbidity or 28-day mortality. ${ }^{6}$

IVIG provides modulatory antibodies that regulate the immune response and resolve any inappropriate immune activation and inflammation and also provides neutralizing antibodies to superantigens that exerts strong antitoxin effect with immunomodulatory consequences. ${ }^{7,8}$ 
Moreover, in the literature it is also reported the use of immunoglobulin A ( $\operatorname{IgA})$ and enriched immunoglobulin $\mathrm{M}(\mathrm{Ig} \mathrm{M})$ in cardiac surgery patients. In a study by Buda et al., the administration of polyclonal IgM-enriched immunoglobulins in septic patients after cardiac surgery resulted in a significantly improved survival rate in the subgroup of patients with severe sepsis. ${ }^{9}$ Similarly, in a study by Friedrich et al., patients received prophylactic an IgMenriched immunoglobulin and their results supported the prophylactic use in patients undergoing procedures with CPB. ${ }^{10}$ Finally, in a study by Kress et al., anergic patients undergoing cardiac surgery received IgA and IgM-enriched immunoglobulin preparation and significantly reduced the incidence of postoperative infections. ${ }^{11}$

The variables used in the method section were taken with some modifications from other studies done in the past. ${ }^{1,5}$ Our laboratory's lowest normal IgG level was $7 \mathrm{~g} / \mathrm{l}$, so this is the reason why this cut-off value of $7 \mathrm{~g} / \mathrm{l}$ for low early postoperative IgG levels was chosen. Moreover, based in the literature pulmonary dysfunction and multiple failure syndrome were associated with levels of IgG less than $7 \mathrm{~g} / .^{5}$

Finally, it was reported that significant IgG levels changes in connection with cardiopulmonary bypass tend to occur with heparin induced thrombocytopenia. ${ }^{12}$ However, in this present series no patient was diagnosed with heparin induced thrombocytopenia or developed any severe postoperative thrombocytopenia.

\section{Study limitations:}

This study had some limitations. 1) The study analyzed only patients electively or semi-electively operated and only the early postoperative IgG levels were obtained. 2) The study population was only 99 patients and only 87 had an on-pump procedure and it may be not sufficiently large group of patients to answer the question related to the role of CPB on IgG. 3) The population was very heterogeneous. Operations that require a cardiotomy may have completely different levels of inflammatory markers than do operations where only the surface of the heart is being touched, i.e. CABG operations.
Based on these limitations a future investigation with a larger homogenous population of patients who would also include acutely ill emergently operated patients should be done. For the study of $\mathrm{CPB}$ related questions, $\mathrm{CABG}$ operations tend to have a more homogenous population with more comparable outcomes.

\section{Conclusion:}

In conclusion, in the literature few studies tried to establish any association between the role of the $\mathrm{CPB}$, the low early postoperative IgG levels and the postoperative complications in cardiac surgery patients. In this pilot study we tried to explore the role of $\mathrm{CPB}$ on the early postoperative levels of IgG and to establish any correlation with the postoperative outcome of these patients. The use of CPB and its duration did not prove to be risk factors for low early postoperative IgG levels. In our opinion the prophylactic use of IVIG in this group of patients has no benefit.

\section{References:}

1. Rankin JS, Glower DD, Teichmann TL, Muhlbaier LH, Stratton CW. Immunotherapy for refractory pulmonary infection after adult cardiac surgery: immune dysregulation syndrome. J Heart Valve Dis 2005: 14:783-791.

2. Lante W, Franke A, Weinhold C, Markewitz A. Immunoglobulin levels and lymphocyte subsets following cardiac operations: further evidence for a Thelper cell shifting. Thorac Cardiov Surg 2005: 53(1):16-22.

3. Siminelakis S, Bossinakou I, Antoniou F, Pallanza Z, Tolios J, Vasilogiannakopoulou D, et al. A study of the effects of extracorporeal circulation on the immunologic system of humans. $J$ Cardiothor Vasc An 1996: 7:893-898.

4. Szerafin T, Niederpold T, Mangold A, Hoetzenecker K, Hacker S, Roth G, et al. Secretion of soluble ST2possible explanation for systemic immunosuppression sfter cardiac surgery. Thorac Cardiov Surg 2009: 57(1):25-29.

5. Rankin JS, Oguntolu O, Binford RS, Trochtenberg DS, Muhlbaier LH, Stratton CW. Management of immune dysfunction after adult cardiac surgery. J Thorac Cardiovasc Surg 2011: 142:575-580.

6. Werdan K, Gunter P, Muller-Werdan U, Enriquez MM, Schmitt DV, Mohr FW, et al. Immunoglobulin G treatment of postcardiac surgery patients with scoreidentified severe systemic inflammatory response syndrome-The ESSICS study. Crit Care Med 2008: 36(3):716-723. 
7. Papageorgiou AC, Acharya KR. Microbial superantigens: from structure to function. Trends Microbiol 2000: 8:369-375.

8. McCormick JK, Yarwood JM, Schlievert PM. Toxic shock syndrome and bacterial superantigens: an update. Annu Rev Microbiol 2001: 55:77-104.

9. Buda S, Riefolo A, Biscione R, Goretti E, Cattabriga I, Grillone G, et al. Clinical experience with polyclonal IgM-enriched immunoglobulins in a group of patients affected by sepsis after cardiac surgery. $J$ Cardiothor Vasc Anesth 2005: 19(4):440-445.

10. Friedrich I, Silber RE, Bauman B, Fischer C, Holzheimer RG. IgM-enriched immunoglobulin preperation for immunoprophylaxis in cardiac surgery. Eur J Med Res 2002: 177(12):544-549.

11. Kress HG, Scheidewig C, Schmidt H, Silber R. Reduced incidence of postoperative infection after intravenous administration of an immunoglobulin M-enriched preperation in anergic patients undergoing cardiac surgery. Crit Care Med 1999: 27(7):1281-1287.

12. Cines DB, Rauova L, Arepally G, Reilly MP, McKenzie SE, Sachais BS, et al. Heparin-induced thrombocytopenia: an autoimmune disorder regulated through dynamic autoantigen assembly/disassembly. J. Clin. Apheresis 2007: 22(1):31-36. 